\title{
Current Status of the Dental Health Care System in Japan
}

\author{
Hirofumi Sasai
}

Department of Oral Medicine, Nihon University School of Dentistry at Matsudo, Matsudo, Chiba 271-8587, Japan

Correspondence to :

Hirofumi Sasai

E-mail : sasai@mascat.nihon-u.ac.jp

Keywords :

social security, dental health care system, reform of medical care system, dental health care community

\begin{abstract}
Facing a major reform of the medical care system, the status of social security in Japan does not have an optimistic outlook. Among the wide -ranging components of the social security system, the medical care system faces serious problems. The national government is beginning to propose legislation to reform Medical Service Laws, review the economic situation relating to medical insurance, and establish comprehensive steps for support through taxation and public financing. Pressing issues specific to the dental community include the supply of and demand for dentists, a reduction in dental health care expenditures, and the reservation of a revised source of funds. Individual dental clinics currently face problems such as reducing the number of patients, and maintaining a balance between revenue and expenditure. Fundamental solutions to these problems can be found only by radical self-reform of the dental system and the dental community. In the future, dentists will need to establish their significance as consulting doctors providing consultations on a regular basis, rather than the present situation in which patients visit the clinic for the first time after experiencing deterioration in their oral condition. To foster a dental health care community that takes the lead in the medical care system of the $21^{\text {st }}$ century, it is necessary to maintain the favorable, though tense, relationship with administrative and political figures, while gaining the consensus of the public.
\end{abstract}

\section{Introduction}

In the face of major reformation of the medical care system, the status of social security in Japan does not have an optimistic outlook. The unprecedented economic slump following the collapse of the so-called speculative "bubble" economy in the early 1990s and aggravating factors such as the resulting deflation have caused a reduction in prices. Further, GDP in fiscal 2001 dropped to the 1995 level, while the ratio of overseas production is showing an increasing trend in manufacturing industries (1). Although recent signs of economic recovery have been detected, the strengthening of international competitiveness in the private sector and a continued increase in individual consumption are indispensable in bringing about a full-scale recovery.

Problems for the federal government include the prediction of future increases in the national contri- bution rate due to ballooning social security expenses, and the overall loss of faith in the system itself (Fig. 1). These problems cannot be solved by short-term measures, but rather by fundamental

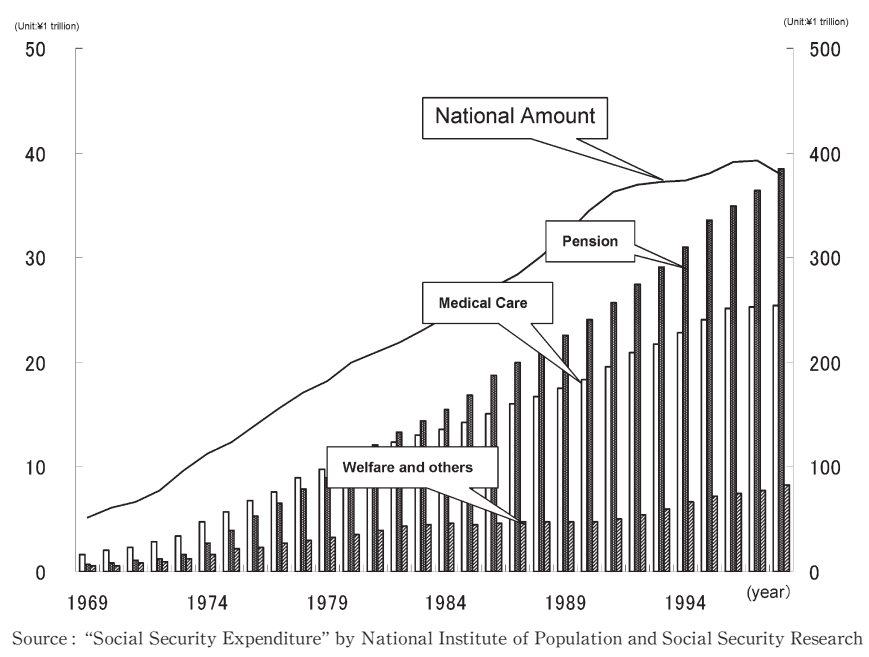

Fig. 1. Changes in the Social Security Benefit Expenditure 
solutions based on a medium to long-term perspective. In other words, it is important to restructure the Japanese social security system, which was established during the years of steep economic growth, and to establish a system that is sustainable in terms of the tax and fiscal realities presented by the current economy, as marked growth is not likely in the near future. For its survival, the social security system must find an adequate financial source, and its greatest challenge is to balance the respective burdens and benefits of the social security system. Since the social security system is a mechanism by which society as a whole supports individuals at potential risk, it must focus on stabilizing the livelihood of individuals who constitute the society. To achieve this, it is necessary to change the sense of values in individual people.

Among the wide-ranging components of the social security system, the medical care system faces serious problems. A variety of factors are intricately interconnected, such as the long-term economic slump, the aging of Japanese society at a faster rate than any other in the world, increasing complexity and diversification of diseases, advancements in medical technology used in treatment of disease, and increasing patient awareness regarding their needs and cost performance. As a result, a satisfactory solution cannot be attained by simply reforming the system without a specific purpose. Medical care service practitioners fully understand that insurance providers cannot become sound and robust within a short period of time without such measures as the Act for Partial Revision of National Health Insurance Laws, implemented in 2003 (2). However, in the short term, the increasing burden on individual patients has resulted in a temporary reduction in the consultation rate, which has led to disease aggravation and a decrease in income for medical care service practitioners.

In order to achieve a functional and sustainable universal medical insurance system, it is impossible to pass the present system to the future generation without making fundamental reforms. Now is the time for both the general public and medical care practitioners to consider what must be done to maintain the system in the face of the collapse of similar systems in other countries.

The Ministry of Health, Labor, and Welfare of Japan presented their analysis of the present status of medical care service in a paper entitled Medical Care Service in the $21^{\text {st }}$ Century as a part of the Draft Proposal of Medical Care System Reform (3). It was noted in the paper that Japan has attained the highest average life expectancy and healthy life expectancy in the world, because of not only improvements in general living standards and public hygiene, but also the efforts of medical care practitioners to create, under the auspices of the national health insurance system, an environment of free access to all medical institutions (Fig. 2). The Ministry's draft proposal also clarified many problems in view of background issues such as declining birth rate, rapid aging of Japan's population, advances in medical technology, and the changing awareness of the public. The Ministry advised that review of the current system must be carried out from multiple perspectives and address the efficiency of the medical care service system, the non-competitive nature of the system, the assurance of reliable medical care, modernization, and the promotion of an efficient intelligence infrastructure common to all medical care services, among other issues. It is expected that the present environment surrounding medical care will quickly change in the near future.

In the revision of the above-mentioned National

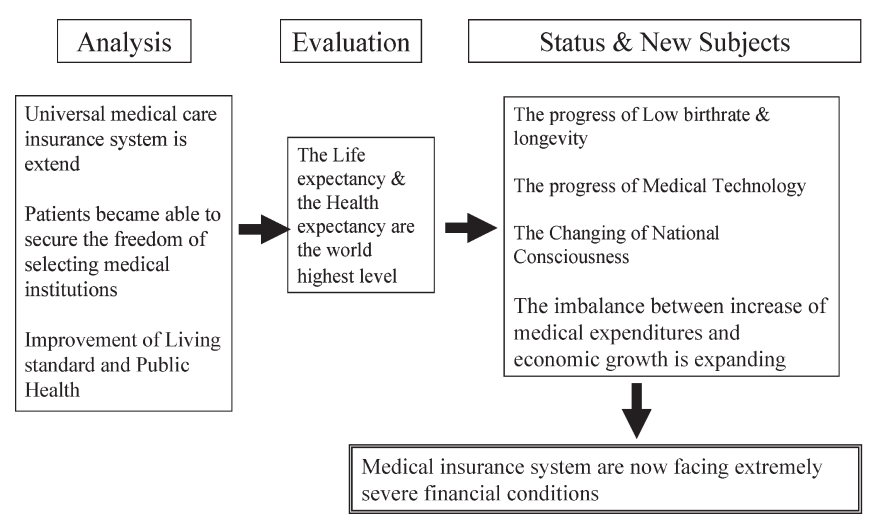

Fig. 2. Current Status and Subjects Health Care System in Japan 


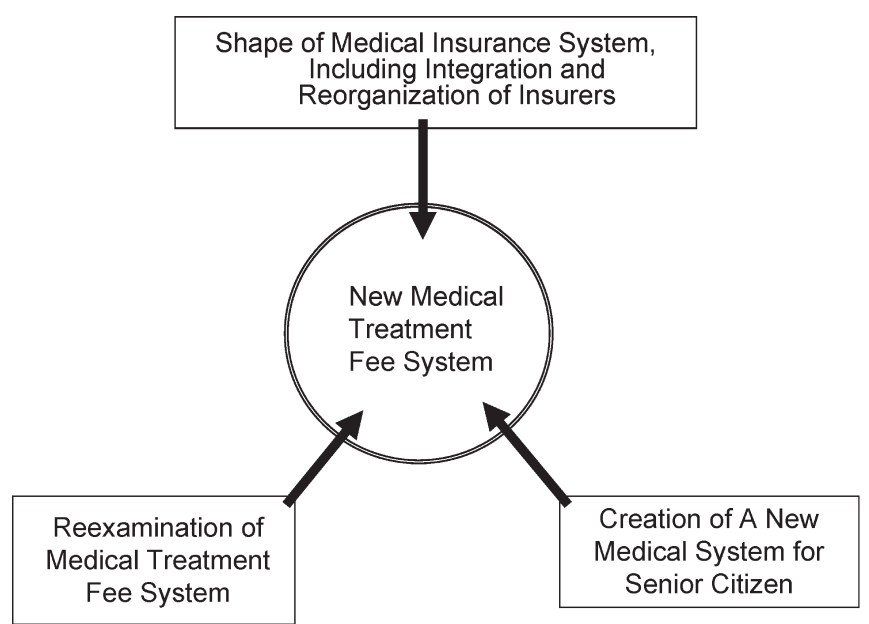

Fig. 3. Structures of reform of Medical Insurance System

Health Insurance Law approved in July 2003 (3), the government outlined basic policies addressing the following : 1) shape of the medical insurance system, including the integration and reorganization of insurers ; 2) creation of a new medical care system for senior citizens; and 3) reexamination of the medical insurance system (Fig. 3). The procedures required for reform of the medical care system are on a deadline. Any revision of the system that necessitates revision of the laws must be started now with a target for completion of 2006 to 2008 , which will be followed by total reform of the insurance system, which began with a revision of medical fees in 2004 (4).

\section{Response of the dental community}

In view of these social changes, how should dental health care practitioners, respond? The national government is beginning to propose legislation that will reform the Medical Service law, review the economic situation of medical insurance, and establish comprehensive steps for support through taxation and public financing. However, there are pressing issues specific to the dental health care community, including supply of and demand for dentists, reduction in dental health care expenditures, and reservation of funds for the revision of the medical fee system. Each dental clinic also currently faces problems such as a reduction in the number of patients, and difficulty in maintaining a balance between revenue and expenditure. Have these problems been caused incidentally or as inevitable consequences of mishandling in the past? Regrettably, the answer is the latter. While the social security system has been making a transition without responding to societal changes since the period of high economic growth, the dental community has also failed to adapt adequately to the changing social situation surrounding dental health care service.

Fundamental solutions to these problems can be established only through radical self-reform by the dental system and community. It is necessary to redefine basic dental health care policy by changing the general public's prejudice and awareness of dentistry and creating an understanding that the purpose of dental health care is to cure dental caries and periodontal disease, extract teeth, and prepare dentures. Reconstructing the relationship between patients and dentists in the community will establish an environment in which dentists can practice their medical business at ease. In addition, the dental community must strive to create an environment for developing innovative dental materials and technologies, even though advanced technical innovation is relatively difficult to obtain. Another pressing issue is the ability of dentists to cope with computerization and the Personal Information Protection Act.

It is understood that the current problems in the dental health care system are caused by a collapse in the balance between supply and demand. As recommended in the final proposal of the 1986 Committee Meeting on Future Supply of Dentists (5), the number of new dentists entering the field was reduced by at least $20 \%$ in 1995 . However, annual transition of the number of dentists continues to increase in spite of the proposal (6) (Fig. 4). Analysis is required to ascertain the necessary increase in the number of dentists, the ideal ratio of dentists to population, and the number of patients; however, it is difficult to determine an optimum level of supply and demand. Kikuchi (7) proposed that the optimum number of patients handled by a single dentist is 23.0. Further, he speculated that the point of excess in supply of 


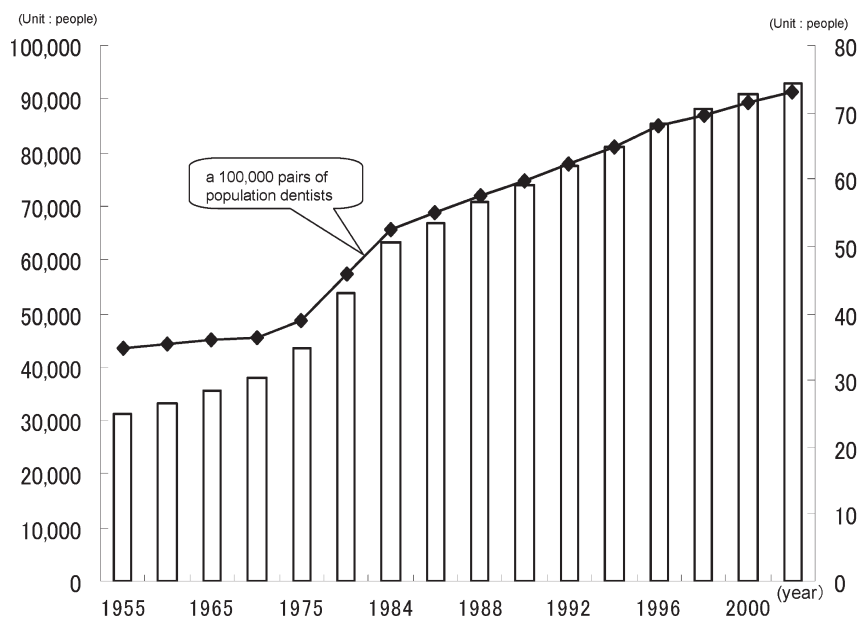

Fig. 4. Annual Transition of the Number of Dentists in Japan (Reference 6)

dentists fell in 1989, which was the mid-term between 1984, when the number of dentists exceeded the proper levels, and 1994, the point at which excess supply of dentists began to increase. Kikuchi calculated the optimum number of patients on the basis of dental care expenditures used as a monetary expression of dental care demand. If the rate of increase in dental care expenditures continues to surpass the rate of increase in number of dentists, the situation is not problematic. However, dental care expenditures will not increase, because the incidence of dental disease is decreasing. In addition, the annual increase in the number of dentists is greater than $2 \%$ at present, which is approximately 10 times greater that the rate of population increase. Therefore, to keep pace with the coming Age of Population Decrease, the number of dentists should be reduced.

The problems associated with an unequal distribution of dentists have been frequently mentioned. However, since an optimum level is not specified, it is merely cited as a problem typical in prefectures where dental colleges are located. It was previously reported that the presence of a dental college increased local information concerning dental health care and enhanced the demand of the community residents in cooperation with the dental care facility (8). The follow-up report prepared in cooperation with the study group found interesting results regarding the relationship between dental care expendi-

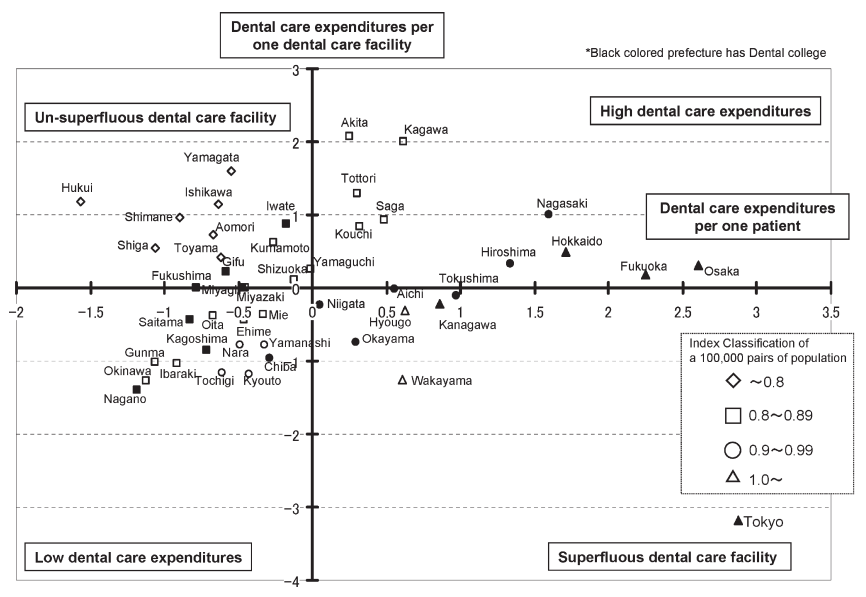

Fig. 5. Regional Differences in Dental care Facilities \& Expenditure (year 2001) (Reference 9)

tures per one dental care facility and per one patient in each prefecture (9). In comparison to the Tokyo area, levels of dental care expenditures per one dental care facility were maintained in such prefectures as Hiroshima and Osaka, and the number of dental institutions was not found to be in excess. Where the number of dental clinics per 100,000 people was high and several dental colleges were located, dental care expenditures per capita were also high (Fig. 5).

The mandatory retirement system for health insurance doctors is being discussed as a possible means to reduce the number of working dentists. Arguments in favor of this system, for not only the dental health care system but also the health insurance system in general, frequently cite the quality of dental health care service provided; however, any speed-beforequality conclusions should be avoided. In Japan, a source of income for dentists cannot be found outside of dental health care expenditures, which are only attainable from medical fees under the medical insurance system. Therefore, a means of increasing financial security must be sought during reformation of the medical insurance system.

As reform moves forward, it will be important to avoid exaggerating problems and postponing solutions. The dental community should set a time schedule and adopt measures to reconstruct a dental environment full of vitality, in which dentists themselves 
can realize new growth and affluence.

It is expected that reforms will give equal importance to the function of each type of medical institution, including specialized medical care clinics in large-scale hospitals, and initial medical care in clinics and mid- and small-sized hospitals. As increased emphasis is placed on the perspective of the patient, the response of dentists to the needs of patients must be a point of reference in the evaluation. It has been said that family or regular dentists play the greatest role in dental health care services. The relationship between patient and dentist first and foremost begins at the initiation of dental disease. In the future, dentists will need to establish their significance as consulting doctors by providing consultation on a regular basis. This is different from the present situation in which patients visit the clinic for the first time after experiencing deterioration in their oral condition. Such consulting doctors will need to acquire the ability to provide primary care, properly treat common diseases, and respond to emergency situations. Emergency pediatric service is not only a part of medical care service. For example, assurance of a reliable and accessible medical care system for emergency dental service, such as treatment of a sudden toothache in a child, will provide a sense of permanent security for parents who worry about childcare in a society with a declining birth rate. Thus, a sustainable relationship with patients can start with easy-to-access daily preventive treatment by family dentists.

Meanwhile, the dental needs of the rapidly increasing elderly patient population for such treatments as artificial dentures must be responded to by lengthy and detailed care. Further, for elderly patients in need of nursing care, detailed visitation care is desirable.

It is expected that the need for aesthetic dental care, which is not regarded as treatment at present, might expand further as the discussions regarding mixed dental care service progress. Dental care professionals will need to handle these types of treatments using the latest available techniques.

\section{Restructuring of the medical insurance system}

Inevitably, it is impossible to manage all of the above-stated problems under the present insurance system. It is natural for medical practitioners to want to establish stable and efficient management procedures for their business. Since medical care income is primarily dependent on the national medical insurance system, reconstruction of the medical insurance system is an important key to solving the present problems. To date, the system of medical insurance has been revised without changing the allocated source of funds, by modifying the score of each dental care service based on its frequency ratio as a component of all dental care expenditure. However, it is nearly impossible to evaluate technical fees accurately with this revision. For example, treatments applied frequently, such as root canal procedures, may occupy the major part of the revised distribution of funds, even if its score is raised only by 1 point. In contrast, low frequency treatments such as oral surgery exert almost no weight on the total amount of dental care services, even if 100 points are added to its score. Those who were involved in the revision understand this situation; however, we are now faced with a dilemma, as the revision might damage the proper means of evaluating the technical fee.

The present method of evaluation by score is the result of a long history of revision and is a compromise solution used to determine the allocation of a limited source of funds. Even if the score for a technique is doubled, it cannot be said that it has attained the proper evaluation.

The scoring method is an evaluation based on the combination of materials and methods used for a medical treatment. However, not until the revision was completed in 2000 was a formalized distinction made between the two. As a result, the funds used for special dental metals can be calculated separately every 6 months during scheduled revisions, independent of regular revisions. Thus, we must make a fundamental review of the methods used to evaluate dental technical fees.

The insurance fee system can be reviewed from 
three perspectives. Remuneration of doctor's fees is paid at a piece rate, based on the difficulty of the technique, while taking into account the time and skill necessary to complete the procedure. Based on the assumption that new technologies will be introduced, each new technique must be objectively evaluated and reevaluated by analysis of the time required, and difficulty of the service provided.

Presently, societies for each medical discipline are in competition with one another as they reformulate the scoring system within the allowable range of revision. This process advances the accumulation of research and objective data for each specialized medical technique. The dental community is currently approximately 10 years behind the medical community in this effort.

These survey results, combined with analysis of the cost of management and facilities to the medical institution, can establish a comprehensive means of evaluation that accurately reflects the cost of a procedure to each medical institution (hospital-feelike factor). Proper evaluations require clarity and transparency regarding cost, as the understanding of society cannot be obtained by appealing only to the loss-making principle of cost accounting as done in the past. The dental community as a whole should defend the necessary cost arrived at, however, coherent reasoning is also indispensable, as the adequacy of the cost will be verified. In certain cases, we should be prepared to make some compromises.

A proper evaluation must also place importance on allowing patients to upgrade dental care based on their preferences, and review the specified treatment expenditure system in view of the diversity of patient needs and the available advancements in medical technology. In an effort to expand the range of dental care expenditures, potential demand might be actualized by making use of the specified treatment expenditures system along with existing courses of medical treatment. As the concept of disease changes over time, attempts should be made to consolidate and clearly specify the therapeutic techniques used for aesthetic recovery, which will gradually gain in popularity mainly among younger patients.
With regard to the proposed review of dental insurance based on medical insurance policy, the following points are important to consider. The maintenance and enhancement of oral function should involve evaluation in accordance with the function and cooperation of dental clinics, departments of dentistry in hospitals, and home dental care services that places emphasis on the prevention of severe dental caries and periodontal disease, as well as coordination with community medical services. Notably, this evaluation places emphasis on the prevention of severe dental caries and periodontal diseases. If the goal of prevention is incorporated into the evaluation of therapeutic techniques, the elimination of existing medical care insurance and the creation of daily consultation and management

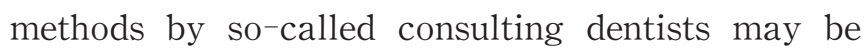
achieved. Accordingly, it will be necessary to universalize the techniques employed in the continuous management of dental caries and the maintenance of periodontal diseases, so as to prepare for the evaluation of these methods. Currently, this is the only proposed method for establishing new opportunities for the survival of the dental health system.

Future revision of the medical treatment fee system should consider the following points : 1) reservation of insurance funds sources necessary for the maintenance of current medical care services combined with unrestrained medical care techniques resulting from technical improvements (including the expansion of the specified treatment expenditures), and 2) establishment of a medical insurance system that evaluates detailed care based on the difficulty of treatments, and not on the number of consultations (evaluation of time and difficulty).

In the future, dentists should be qualified with the skills of a medical practitioner. At present, though nearly all of the students in dental colleges aim to become a practicing dentist, they cannot study business administration or management in college and graduate school. It is important to provide the opportunity for business education study to those who want to be a practitioner or manager of a dental clinic. Additionally, providing extension courses or 
partially opening college business and management lectures to those who have already engaged in dental management must be discussed. Under the present circumstances, such as the rise in cost of commercial health insurance and the new entry of business corporations into the medical care service industry, it is not difficult for service providers who have overcome free competition to outstrip those based in the universal medical care insurance system. Also, there is almost no ground for asserting that their entry into medical care service might downgrade these services. Thus, it is necessary to expand the range of business education concerning dental clinic management in colleges and graduate schools. For education programs regarding medical management, it is desirable to make use of medical practitioners who are experienced in the operation of such business. In undergraduate school, students should acquire necessary administrative skills, such as solution-finding skills, self-reliance, and determination.

\section{Correspondence to the new field}

To revive the dynamism of the dental community, dentistry should be converted to a field in which dentists are active in business and have a diversified sense of values, free of the concept that dentist equals practitioner. To achieve this, it is necessary to establish the sense that dentists are responsible for the public sector of dental health care services that should be understood and valued by the public.

As suggested in the Health Promotion Law, oral health consists of not only medical care but also health maintenance and promotion. Though these components have not been evaluated adequately, it is important to construct a system with varied fields, in which dentists are urged to fully use and improve their skills and are evaluated properly.

Innovation in dental care techniques is another requisite. New materials offering lower prices and improved operation are indispensable for the development of dental health care. The development and promotion of new dental instruments can enhance safety, as well as develop and extend therapeutic technology in interdisciplinary fields. Dental colleges are clearly promising sources of dental health care service with high technology and competent personnel, and should contribute to the advancement of dental health care through the creation of new technologies and the discovery of new findings. It is expected that their research activities will be activated by the formulation of cross-sectional, inter-college study groups mediated by the society of dentistry. It is also advisable for related organizations to establish an incentive system by which research expenses are allotted to researchers for not only research on the development of new technology, but also for those engaged in material and clinical epidemiological studies.

In the effort to maintain and promote a healthy and long living society, knowledge of the human genome will be utilized routinely for disease treatment and prevention, ranging from research to industrialization and application to society. In the field of disease treatment, genetic information is applied to creating medicines and providing diagnoses, while in the field of disease prevention this information is utilized for analyzing the effect of environmental factors on the human body. Even in the field of dentistry, it is expected that genetic information might be useful for developing therapeutic technology and materials, as a new perspective for studies in collaboration with researchers in other fields.

Information technology in the field of health care service is also an important item, especially from the standpoint of business management. The basic design for computerization has been formulated, and the actual procedures and methods to accomplish the goals of the 5-year computerization plan started in 2002 have been established. To simplify the introduction of an electronic medical chart system, appropriate terms, codes, and forms must be standardized. For medical insurance, billing by the use of magnetic media is also being promoted. An efficient remuneration system can be promoted by establishing a system to be shared among medical institutions, organizations in charge of probation and payment, and insurance companies. In the future, an on-line billing system will be constructed, and nearly all dental 
institutions will be covered following enactment of the Personal Information Protection Act in April 2005. The dental community as a whole should positively comply with this legislation, while acquiring subsidies by making use of the variety of systems related to it.

With regard to a clinical training system for the purpose of fostering and providing personnel for dental care service, it is necessary to establish a program that improves the standards of dental health care and continuously enhances available technology. Clinical training for the medical profession became compulsory in fiscal 2004, though latent problems will be gradually exposed. Clinical training for the dental profession will become compulsory in fiscal 2006 and the basic policies of the system will be similar to those for medical doctors. However, in the field of dentistry, where clinics in a variety of styles will inevitably be used as major training sites, the establishment of a dentistry specific system and flexible execution of the system are necessary.

Economic and systematic factors have resulted in the need for improving dental care standards in developing countries. In the Asia-Pacific region, of which Japan is a member, the system for dental health care has slowly been demonstrating improvement. However, in some countries and regions, the system has fallen far behind due to economic and systematic reasons, and Japan must take the initiative in providing academic and medical support for these nations. Such activities by dentists should be publicized so as to make the public aware that dentists are cultivating the base of social valuation. Further, to continue the overseas dental health care activities currently carried out mainly by dental colleges, necessary requests should be made to secure official support.

\section{Partnership with other concerned parties}

In an attempt to realize the goals of the abovementioned discussion, it is important for the Japan Dental Association to foster a close relationship with administrative and political figures. In the recent scandal involving the Central Social Insurance Medi- cal Council, a deliberative body that should be open and fair lied and applied old-fashioned problem solving methods. The dental community as a whole must share responsible for the egoism of this runaway part of the association.

To foster a dental health care community that takes the lead in the medical care system of the $21^{\text {st }}$ century, it is necessary to maintain its favorable, though tense, relationship with administrative and political figures, while gaining the consensus of the public. To maintain this relationship and gain public support, the dental community itself should make clear and specific proposals that reflect the opinions of the dental health care service participants. Securing competent personnel who can conduct serious policy discussions regarding dental health care service in cooperation with think-tanks, scientific societies, and universities will allow the dental community to gain influence by collecting information regarding the policies of central and local governments, and analyzing them from a varied perspective.

\section{References}

1. Japan Federation of Economic Organizations, Towards a society where everyone can demonstrate the entrepreneur spirit. Document JFEO, Tokyo, Japan, Spring, 2002.

2. On the basic policy based on Article 2-2 of Supplementary Provisions to the Act for Partial Revision of National Health Insurance Laws, endorsed by the Cabinet, March, 2003.

3. Ministry of Health Labour and Welfare, Medical Care System in the $21^{\text {st }}$ Century. Document MHLW, September, 2001.

4. Ministry of Health Labour and Welfare, Reexamination of Medical Treatment Fee System (Proposal by Ministry of Health Labour and Welfare). Document MHLW, September, 2001.

5. Ministry of Health and Welfare, Dental Health Division, The examination committee's report of the supply and demand in the future dentists. Document MHW, July 21, 1986.

6. Statistics and Information Department, Minister's Secretariat, MHLW, Survey of Physicians, Dentists and Pharmacist. Statistics and Information Department, Minister's Secretariat, MHLW.

7. Kikuchi T, Miyatake K, Kamagata K, Umemura O, 
Horiuchi H, Kawabuchi K, Igarashi T, Okamoto E, Ishimoto $\mathrm{T}$, Itou $\mathrm{M}$ : White report on Dental Health Care System, Japan Dental Association, 34-40, 2003.

8. Okada M, Yamada Y, Okawa Y, Takahashi Y, Murai M, Miyatake K, Ishii T: An Analysis of Regional Differences in Dental Care Facilities and in Dental Care Expenditure Part 2: Jpn J Dent, 38: 310-320,
2004.

9. Sasai H, Okada M, Yamada Y, Sato H, Okawa Y, Takahashi Y, Murai M, Miyatake K, Ishii T: An Analysis of Regional Differences in Dental Care Facilities and in Dental Care Expenditure Part 3: Jpn J Dent, 39 : 344-351, 2005. 